\title{
Ansiedade pré-competitivas em basquetebolistas da selecção nacional de sub-16
}

\section{Pre-competitive in basketball players of the under-16 national team}

\author{
V. Tembe, C. Dezanove, P. Saveca, H. Machoi, F. Pacheco
}

ARTIGO ORIGINAL | ORIGINAL TITLE

\begin{abstract}
RESUMO
A ansiedade é uma resposta fisiológica ao momento vivenciado que seja doloroso, pois ambos possuem conexão, a dificuldade vivenciada e a ansiedade de superá-lo. O estudo teve como objetivo analisar as características de factores de ansiedade pré - competitiva nos jogos da selecção nacional de Moçambique Sub-16. A amostra foi constituída por 12 atletas, com idades compreendidas entre os 14 - 16 anos de idade dos quais 4 com 16 anos e 8 com 14 anos. O instrumento utilizado foi a "Lista de Sintomas de Stress e Pré-competitiva (LSSPC) desenvolvida por Chagas (1995). Os resultados evidenciaram níveis moderados de ansiedade pré-competitiva nos seis jogos disputados com soma média de 56,92; os seis jogos disputados, as basquetebolistas apresentaram, respectivamente, a valorização da ansiedade somática com valores seguintes: 59,16;53,34; 59; 61,5; 56,17 e 55,4. As atletas valorizaram, relativamente, a ansiedade somática nos seis jogos com os valores seguintes: 3,$71 ; 3,09$; 3,82; 4,12; 3,58 e 3,69. As atletas da selecção de basquetebol participantes no Campeonato Africano de Basquetebol sub-16 em 2017 foram dominadas pela ansiedade moderada com predominância do factor somático.
\end{abstract}

Palavras-chave: basquetebol, adolescentes, ansiedade

\begin{abstract}
Anxiety is a physiological response to the painful experience because both have a connection, the difficulty experienced and the anxiety to overcome it. The study aimed to analyze the characteristics of pre-competitive anxiety factors in the national team of Mozambique U16. The sample consisted of 12 athletes aged $14-16$ years, of which 4 aged 16 and 8 aged 14 years. The instrument used was the "List of Stress and Pre-Competitive Symptoms (LSSPC) developed by Chagas (1995). The results showed moderate levels of pre-competitive anxiety in the six games played with an average sum of 56.92; In the six games played, the basketball players presented the valorization of the somatic anxiety with the following values: $59.16 ; 53.34 ; 59 ; 61.5 ; 56.17$ and 55.4 . The athletes valued somatic anxiety relatively in the six games with the following values: $3.71 ; 3.09 ; 3.82 ; 4.12 ; 3.58$ and 3.69 . Basketball players participating in the 2017 African Under-16 Basketball Championship were dominated by moderate anxiety with a predominance of the somatic factor.
\end{abstract}

Keywords: basketball, adolescents, anxiety

Submitted: 05.28.2020 | Accepted: 07.24.2020

Vicente A. Tembe, Carlos Dezanove, Hermenegildo Machoi. Universidade Pedagógica, Faculdade de Educação Fisica e Desporto, Maputo, Moçambique.

Paulo T. Saveca, Fernando Pacheco. Universidade Eduardo Mondlane, Maputo, Moçambique.

Endereço para correspondência: Vicente A. Tembe, Universidade Pedagógica de Maputo - Faculdade de Educação Física e

Desporto, Av. Eduardo Mondlane 955, CP. 2107- Maputo-Moçambique.

e-mail: vicenteatembe@gmail.com 
A prática do basquetebol no contexto das seleções nacionais para além de ser um momento de glorificação, é desde há muito tempo considerada um factor de exaltação de uma determinada comunidade ou país. Relegados que são os aspectos de benefícios físicos, psicológicos e sociais na prática de desporto de alta competição.

As seleções nacionais fazem parte da preocupação duma dada sociedade o que faz com os seus integrantes estejam expostos a pressões com a necessidade de demonstrar resultados que provoque a alegria comum. Este facto requere que se controle os níveis da ansiedade no geral e da ansiedade pré -competitiva em especial. Aliás, os desejos dos adultos tendem, por vezes a suplantar os níveis de exigência dos próprios executantes provocando níveis de ansiedade imprevisto.

Realmente o que deveria ser prazeroso, como jogar na seleção nacional de basquetebol, também tornou-se um compromisso, uma obrigação de resultado, da qual depende a auto-estima, e na qual o objecto de interesse principal é o produto final: o rendimento. A espontaneidade e a criatividade das brincadeiras cederam seu lugar a actividades dirigidas, cópias fiéis do modelo adulto de ganhar, em que o resultado final é mais importante que todo o desenrolar da actividade meramente educativa e recreativa.

Os jogos entre as seleções nacionais tornaram-se sinónimo para glorificação e engrandece os vencedores como representantes sociais. Existindo a tendência de cobrança de resultados aos jovens selecionados o que requere o controlo do ambiente pré-competitivo onde podemos encontrar a ansiedade em forma cognitiva, somática e autoconfiança que é referenciada por Martens et al. (1990), Machado (2006) e da seguinte forma:

a) Ansiedade Cognitiva consiste em pensamentos negativos e preocupações sobre a performance, incapacidade para se concentrar e atenção disruptiva; b) Ansiedade Somática é uma percepção das manifestações físicas da ativação, caracteriza da por sensações físicas e

c) A autoconfiança é uma crença geral do indivíduo, que pode realizar com sucesso uma determinada ação e atividade.

No geral a ansiedade é conceituada como um estado emocional que surge em resposta à maneira como a pessoa avalia ou interpreta uma situação, seja em momentos de perigo real ou imaginário, desencadeando sintomas como frio no estômago, opressão no peito, palpitações, transpiração excessiva, dor de cabeça e/ ou falta de ar (Araújo et al., 2007; Gama et al., 2008; Román \& Savoia, 2003).

Dependendo de sua circunstância ou intensidade, a ansiedade pode ser útil ou tornar-se patológica, prejudicando o funcionamento psíquico/somático do indivíduo. Em níveis normais, trata-se de um fenómeno fisiológico responsável pela adaptação do organismo em situações de perigo. Entretanto, quando a ansiedade é excedente, ao invés de contribuir, desencadeia a falência da capacidade adaptativa (Benute et al., 2009).

A ansiedade interfere nos comportamentos e motivos para os indivíduos para praticarem desporto. Podendo também desempenhar um papel fundamental na determinação dos resultados como onde e com quem as pessoas se exercitam como é o caso de um jogo de basquetebol (Spink, 1992).

Nos estudos de ansiedade pré-competitivo, um contributo assinalável foi feito por Martens em 1977, ao sugerir o Sport Competition Anxiety Test (SCAT) e, mais tarde, o Competitive State Anxiety Inventory-1 (CSAI-1) com objectivo de compreender o ser humano nos momentos pré-competitivos desportivos. Na base do teste supracitado surgiram vários resultados de hierarquização dos factores de ansiedade pré-competitivo cognitiva, somática e autoconfiança de que são exemplos os mencionados no Quadro 1. 
Quadro 1

Factores da ansiedade pré-competitiva

\begin{tabular}{|c|c|}
\hline Autor & Resultados \\
\hline Barbacena \& Grisi (2008) & $\begin{array}{l}1^{\circ} \text { Autoconfiança } \\
2^{\circ} \text { Cognitiva } \\
3^{\circ} \text { Somática } \\
\end{array}$ \\
\hline Bertuol \& Valentini (2006) & Moderada \\
\hline Gotine (2019) & $\begin{array}{l}1^{\circ} \text { Ansiedade Cognitiva } \\
2^{\circ} \text { Ansiedade Auto Confiança } \\
3^{\circ} \text { Ansiedade Somática }\end{array}$ \\
\hline Vasconcelos-Raposo et al. (2007) & $\begin{array}{l}1^{\circ} \text { Autoconfiança com } \\
2^{\circ} \text { Ansiedade cognitiva } \\
3^{\circ} \text { Ansiedade somática } \\
\end{array}$ \\
\hline Santo (2008) & $\begin{array}{l}1^{\circ} \text { Autoconfiança } \\
2^{\circ} \text { Ansiedade Somática } \\
3^{\circ} \text { Ansiedade Cognitiva } \\
\end{array}$ \\
\hline Machava (2019) & $\begin{array}{l}1^{\circ} \text { Autoconfiança } \\
2^{\circ} \text { Somática } \\
3^{\circ} \text { Cognitiva } \\
\text { Moderado }\end{array}$ \\
\hline Madime (2017) & $\begin{array}{l}1^{\circ} \text { Somática } \\
2^{\circ} \text { Cognitiva } \\
3^{\circ} \text { Autoconfiança } \\
\end{array}$ \\
\hline Massingue (2014) & $\begin{array}{l}1^{\circ} \text { Somática } \\
2^{\circ} \text { Autoconfiança } \\
3^{\circ} \text { Cognitiva }\end{array}$ \\
\hline
\end{tabular}

As evidências de valorização diferenciada dos factores de ansiedade pré-competitiva, requere da equipa técnica, por exemplo duma seleção nacional de basquetebol, uma atenção com vista a adotar estratégia para lidar com eles (ansiedade somática, cognitiva e autoconfiança).

De acordo com os pressupostos, o objetivo deste estudo foi analisar as características de factores de ansiedade pré - competitiva nos jogos da selecção nacional de Moçambique Sub-16.

\section{MÉTODOS}

O estudo é de natureza transversal, pois os dados foram recolhidos num único momento temporal. Também é de caracter exploratório e quantitativo pois é orientado para os resultados.

\section{Amostra}

A nossa amostra é constituída por 12 atletas, com idades compreendidas entre os 15 aos 16 anos de idade dos quais 4 com 16 anos e $8 \mathrm{com}$ 15 anos. Esta amostra constitui, por coincidência o número total das atletas ou população da selecção em estudo.

\section{Instrumentos}

O instrumento de recolha de dados utilizado foi um questionário auto administrado, denominado "Lista de Sintomas de Stress e Pré-competitiva (LSSPC)" desenvolvido por Chagas (1995). Este instrumento é composto por dezassete perguntas objetivas, o qual utiliza a escala Likert, abordando itens que incluem a avaliação de sintomas psicológicos, fisiológicos e sociológicos, os quais estão relacionados com a ocorrência de ansiedade pré-competitiva e competitiva. 
Cada pergunta da referida lista teve uma apreciação de 5 alternativas que foram: $1=$ Quase nunca, $2=$ Geralmente, $3=$ As vezes, $4=$ Raramente, 5=Quase nunca. Sendo que, também valorizam em baixa ansiedade (17 a 40 pontos), ansiedade moderada (41 a 62 pontos) e ansiedade elevada (63 a 85 pontos). Os somatórios dos factores podem ser divididos por números de indicadores de cada factor e obter-se por este meio a hierarquia.

\section{Procedimentos}

Para a recolha de informações relacionadas com a elaboração deste trabalho recorreu-se às atletas de basquetebol da selecção nacional onde um membro da Rede de Estudos do Desporto e Desenvolvimento Humano (REDH) fazia parte da equipa técnica na qualidade de treinador adjunto. Contudo, solicitou-se a Federação Moçambicana da Basquetebol a anuência que foi prontamente satisfeita e inclusive indigitada uma psicóloga para acompanhar os trabalhos. Após a sua permissão, foi realizada uma breve explicação acerca da pertinência e aplicabilidade dos questionários às atletas, assim como as normas do seu preenchimento. Contudo, o ambiente de aceitabilidade da pesquisa propiciou a realização de recolha dos dados nos seis jogos. De realçar que, depois do primeiro jogo os próprios atletas exigiam o questionário para responder às questões nas vésperas do jogo. De salientar que a distribuição e a recolha dos questionários foram feitas 1 hora antes da competição com vista a atender os objectivos do estudo.

\section{RESULTADOS}

Os fatores de ansiedade que mais manifestaram-se nas basquetebolistas da seleção sub-16 são: Autoconfiança (no $1^{\circ}$ jogo da vitória) e Somática (nos cinco jogos onde a equipa obteve derrota). No Quadro 2, estão espelhadas as características da valorização da ansiedade pré-competitiva nos contextos jogos e resultados.

Os níveis de ansiedade das basquetebolistas nos jogos demonstram, pela ordem dos jogos, os valores seguintes: $1^{\circ}$ jogo, resultado vitória, com nível de ansiedade de 59.16 e diferença de 11 pontos nas conversões; $2^{\circ}$ jogo, resultado derrota, com nível de ansiedade de 53.34 e diferença de 7 pontos convertidos; $3^{\circ}$ jogo, resultado derrota, com nível de ansiedade de 59 e diferença de 3 pontos convertidos; $4^{\circ}$ jogo, resultado derrota, com nível de ansiedade de 61.16 e diferença de 38 pontos; $5^{\circ}$ jogo, resultado derrota, com nível de ansiedade de 56.17 e diferença de 17 pontos e $6^{\circ}$ jogo, resultado derrota, com nível de ansiedade de 55.4 e diferença de 17 pontos (Quadro 2).

Quadro 2

Valores da ansiedade pré-competitiva

\begin{tabular}{|c|c|c|c|c|c|}
\hline \multirow{2}{*}{ Jogo } & \multirow{2}{*}{$\begin{array}{l}\text { Contextos } \\
\text { Resultados }\end{array}$} & \multicolumn{3}{|c|}{ Factores Ansiedade Pré-Competitiva } & \multirow{2}{*}{ Nível } \\
\hline & & Cognitiva & Somática & Autoconfiança & \\
\hline $1^{\circ} \mathrm{Jogo}$ & Vitória por 38 x 49 & 12.00 & 29.75 & 17.41 & 59.16 \\
\hline $2^{\circ} \mathrm{Jogo}$ & Derrota por $64 \times 57$ & 12.42 & 24.75 & 16.17 & 53.34 \\
\hline $3^{\circ}$ Jogo & Derrota por $61 \times 64$ & 11.67 & 30.75 & 16.58 & 59 \\
\hline $4^{\mathrm{o}} \mathrm{Jogo}$ & Derrota por $33 \times 71$ & 11.58 & 33.00 & 16.92 & 61.5 \\
\hline $5^{\circ} \mathrm{Jogo}$ & Derrota por $60 \times 43$ & 11.58 & 28.67 & 15.92 & 56.17 \\
\hline $6^{\circ} \mathrm{Jogo}$ & Derrota por $39 \times 56$ & 10.75 & 29.57 & 15.08 & 55.4 \\
\hline \multicolumn{5}{|c|}{ Soma } & 56.92 \\
\hline
\end{tabular}


As basquetebolistas de seleção-sub16 atribuíram valores que variavam de 2.68 a 3.1 na ansiedade cognitiva, 3.09 a 4.12 na ansiedade

Gráfico 1

Niveis médios dos factores da ansiedade pré-competitiva somática e 3.01 a 3.48 na autoconfiança (na escala de 1 a 5) conforme o gráfico 1 .

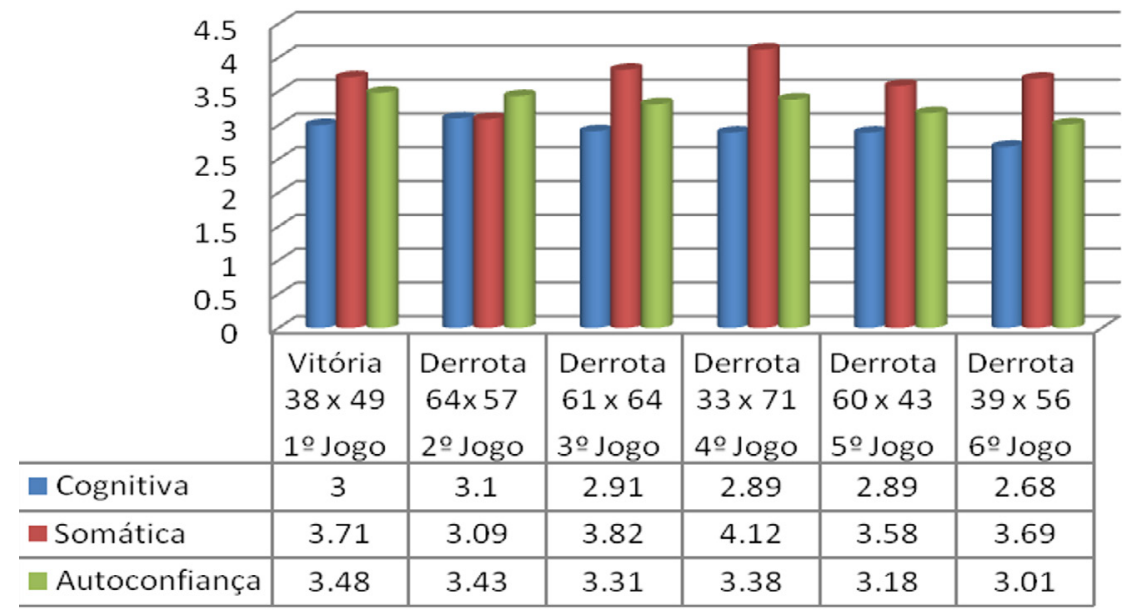

\section{DISCUSSÃO}

$\mathrm{Na}$ interpretação da ansiedade pré-competitiva foi da extrema importância para entender como as atletas interpretavam o significado da conquista, e como as diferenças individuais de cada uma podiam influenciar o processo da conquista.

O nível de ansiedade pré-competitiva, dependendo da soma obtida, pode ser classificado de baixa ansiedade (17 a 40 pontos), ansiedade moderada (41 a 62 pontos) e ansiedade elevada (63 a 85 pontos). As participantes no estudo atribuíram o nível moderado de ansiedade pré-competitiva. Estes resultados corroboram com os resultados de Machava (2019) e Bertuol \& Valentini (2006) na demonstração do nível de ansiedade moderada.

Jogar na selecção tende a apresentar exigências acrescidas o que propicia a ansiedade alta ou moderada. Os níveis de ansiedade apresentada nas competições tendem a diferenciar das apresentadas nos treinos ou seja, nível baixo nos treinamentos e alto nas competições (Becker Jr., 1989). Sendo que, o papel do treinador afigura-se importante para optimizar a ansiedade.
As basquetebolistas da selecção sub-16 demostraram uma relativa valorização da ansiedade somática no período que antecede a competição. Esta evidência corrobora com os estudos de Madime (2017) e Massingue (2014) que nos seus estudos relatam a valorização da ansiedade somática.

A ansiedade somática demonstra um elevado nível de activação que é essencial para actividades desportivas que requerem velocidade, resistência, salto em altura e forca como é o caso de basquetebol. As basquetebolistas da seleção-sub-16 atribuíram valores relativamente superiores à ansiedade somática visto como de ordem fisiológica. Sendo que, a sua activação pode estar relacionada com estímulos relacionados com assistentes nas bancadas, importância do jogo, factor casa.

A segunda dimensão mais valorizada foi autoconfiança o que corroborou com os estudos de Gotine (2019) e Massingue (2014). Na visão de Martens et al. (1990) a autoconfiança deve ser equacionada como a ausência da ansiedade cognitiva. Entretanto, a fraca confiança e confiança excessiva nas capacidades individuais e do grupo podem contribuir para a sua valorização. 


\section{CONCLUSÃO}

Os jogos da selecção nacional de Moçambique Sub-1 foram caracterizados pelo domínio da ansiedade somática e um nível moderado da ansiedade pré - competitiva.

A ansiedade somática deve ser visto como forma de preparação fisiológica e não um construto negativo. Neste prisma pode se considerar como uma activação do organismo para encarar o jogo.

\section{Agradecimentos:}

Nada declarado.

\section{Conflito de Interesses:}

Nada declarado.

\section{Financiamento:}

Nada declarado.

\section{REFERÊNCIAS}

Araújo, S., Melo, M \& Leite, J. (2007). Transtornos de ansiedade e exercício físico. Revista Brasileira de Psiquiatria, 29 (2), 164-171.

Barbacena, M. M. \& Grisi, R.N.F. (2008). Nível de ansiedade pré-competitiva em atletas de natação. Conexões, Revista da Faculdade de Educação Física da UNICAMP, Campinas, v. 6, n. 1,- ISSN 1983 - 9030.

Benute, Gláucia Rosana Guerra et al (2009). Abortamento espontâneo e provocado: ansiedade, depressão e culpa. Rev. Assoc. Med. Bras., São Paulo, v55 n3, p. 322-327. Available from <http://www.scielo.br/ scielo.php?script $=$ sci_arttext $\&$ pid $=S$ $010442302009000300027 \& \operatorname{lng}=$ en \& $\mathrm{nrm}=\mathrm{iso}>$. access on 07 Aug. 2019. http://dx.doi.org/10.1590/S010442302009000300027.

BertuoL, L.; Valentini, C. (1995). Ansiedade competitiva de adolescentes: gênero, maturação, Nível de experiência e

modalidades esportivas. Revista da Educação Física, Maringá, v. 17, n. 1, p. 65-74, 2006. Chagas M.H. (1996). Análise do estresse psíquico na competição em jogadores de futebol de campo das categorias juvenil e júnior. Belo Horizonte: Escola de Educação Física da UFMG, (dissertação de mestrado).

Gama, M. M. A., Moura, G.S., Araújo, R. F., Silva, F.T. (2008). Ansiedade-traço em estudantes universitários de Aracaju (SE). Rev Psiquiatr RS. 2008;30(1):19-24.

Gotine R. L. (2019). Avaliação dos Níveis de Ansiedade Pré-competitiva em futebolistas Juvenis da Cidade da Maxixe. Monografia. Universidade Pedagógica de MaputoFEFD.

Román, S. \& Savoia, M.G. (2003). Pensamentos automáticos e ansiedade num grupo de jogadores de futebol de campo. Psicologia: Teoria e Prática, São Paulo, v. 5, n. 2, p. 13-22, 2003.

Santo, R. J. F. (2008). Competências Mentais e Ansiedade Competitiva em Atletas de Basquetebol. Monografia. Universidade de Coimbra.

Madime, T. (2017). Ansiedade pré-competitiva, burnout e orientação motivacional em atletas da modalidade de natação dos Clubes da Cidade de Maputo. Dissertação. Universidade Pedagógica de Maputo-FEFD.

Machado, A. A. (2006). Psicologia do esporte: da educação física escolar ao treinamento esportivo. São Paulo: Guanabara Koogan.

Machava, A. A. (2019). Ansiedade Pré Competitiva e Orientação Motivacional em Adolescentes Praticantes de Actividades Desportivas no Distrito Municipal Kapfumo. Dissertação- Universidade Pedagógica de Maputo-FEFD.

Martens, R.; Vealey, r. \& Burton, d. (Ed.). (1990). Competitive anxiety in sport. Champaign, In: Human Kinetics.

Massingue, A. A. (2014). Avaliação do stress pré-competitivo e orientação motivacional em jogadores de futebol com idades compreendidas 
$\begin{array}{lllll}\text { entre } & 14 & \text { e } & 22 & \text { anos. Monografia. } \\ \text { Universidade } & \text { Pedagógica de Maputo- }\end{array}$ FEFD.

Vasconcelos-Raposo, J.; Lázaro, J.; Mota, M.;

Fernades, H.; (2007). Caracterização dos niveis de ansiedade em praticantes de atletismo. Motricidade, 3(1): 298-314.
Vasconcelos-Raposo, J. \& Fernandes, H. (2004). Análise confirmatória do CSAI-2. Lisboa: Ed da Universidade de Trás-osMontes e Alto Douro.

Weinberg, R. S. \& Gould, R. (2001). Fundamentos da psicologia do esporte e do exercício. Porto Alegre: Artmed, 560 p. 